\title{
Follicular Carcinoma of Thyroid Metastasizing to the Sphenoid Sinus
}

\author{
${ }^{1}$ Suma R Radhakrishnan, ${ }^{2}$ Asjeena Andru, ${ }^{3}$ Vijayan Peettakkandy
}

\begin{abstract}
Introduction: Metastatic tumors to the nose and paranasal sinuses are unusual with 167 cases published in the literature since 1951. The most common locations of the primary tumors are renal, lung, breast, testis, gastrointestinal tract and thyroid gland. There are only 26 documented cases of metastasis to the sphenoid sinus of which only six are from follicular carcinoma thyroid.
\end{abstract}

Materials and methods: Case reports of two patients referred to our department with ocular symptoms having a final diagnosis of follicular carcinoma thyroid with sphenoid sinus metastasis were studied and literature review done.

Discussion: Metastasis to the sphenoid sinus from follicular carcinoma thyroid is a rare entity and here we present two more cases of which one was an occult thyroid carcinoma. Both patients presented with ocular symptoms and the features of metastasis was the first sign of disease.

Conclusion: Early diagnosis of signs and symptoms relating to metastatic sphenoid disease is necessary as these are frequently the first presentation of malignancy. Even though cure of patients with sphenoid sinus metastasis has not been reported, palliation with resolution of morbidity is possible.

Keywords: Follicular carcinoma thyroid, Paranasal sinus malignancy, Sphenoid sinus metastasis.

How to cite this article: Radhakrishnan SR, Andru A, Peettakkandy V. Follicular Carcinoma of Thyroid Metastasizing to the Sphenoid Sinus. Clin Rhinol An Int J 2015;8(2):53-57.

Source of support: Nil

Conflict of interest: None

\section{INTRODUCTION}

Tumors of the nose and paranasal sinuses constitute $3 \%$ of all head and neck malignancies. Among these, majority are primary tumors. Secondary deposits in the nose and paranasal sinuses are rare; less than 200 cases have been reported to date. ${ }^{1}$ The common primary sites

\footnotetext{
${ }^{1,3}$ Associate Professor, ${ }^{2}$ Junior Resident

${ }^{1,2}$ Department of ENT, Government Medical College, Calicut Kerala, India

${ }^{3}$ Department of Neurosurgery, Government Medical College Calicut, Kerala, India

Corresponding Author: Suma R Radhakrishnan, Associate Professor, Department of ENT, Government Medical College, Calicut, Kerala, India, Phone: 04952358969, e-mail: drsumavijay@gmail.com
}

are the prostate, breast, lung, kidney and the thyroid. ${ }^{2-4}$ Metastasis to sphenoid sinus is exceedingly rare, with only 26 cases reported to date in literature. ${ }^{6}$

The clinical presentation of the metastases in paranasal sinuses is similar to primary tumors in the same location and are frequently the first presentation of disease. Some of the most frequent symptoms are headache, loss of vision, diplopia, facial numbness, loss of hearing, and other symptoms related to single or multiple cranial neuropathies (II to VIII nerves). ${ }^{2,4,7}$ Epistaxis is characteristically associated with metastases from renal cell carcinoma ${ }^{8}$ and thyroid cancer. ${ }^{3}$

\section{CASE REPORTS}

\section{Case 1}

A 62-year-old female presented in our neuromedicine outpatient department (OPD) (Fig. 1) with complaints of headache and double vision of 4 months duration and progressive bilateral visual loss of 1 month duration. The history was negative for pain, redness or protrusion of eyes, numbness of face, nasal obstruction, bleeding from nose, photophobia or vomiting. She had no comorbid conditions except for a huge thyroid swelling, which had been there for the past 27 years and had bothered her the least. General examination was normal except for the huge thyromegaly, more toward the left side of neck. On examining the cranial nerves, perception of light was absent on both sides. In addition, there was bilateral third nerve palsy and right sixth nerve palsy. Rest of nervous

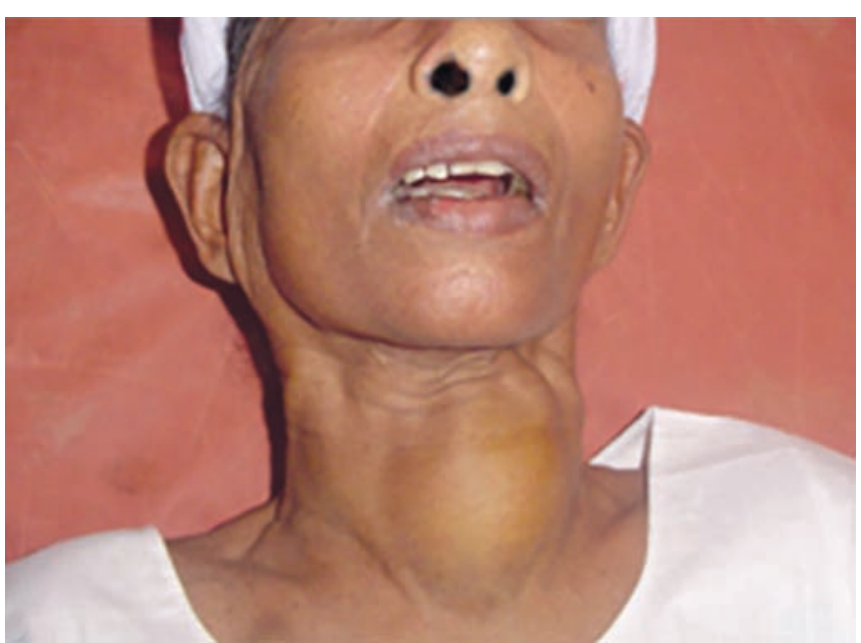

Fig. 1: Patient with huge thyromegaly 
system examination was normal. Fundus examination revealed bilateral optic atrophy. Other systems were within normal limits.

Blood and urine investigations were normal except for an elevated erythrocyte sedimentation rate (ESR). A fine needle aspiration from the thyroid swelling was done which showed follicular cells in a background of blood and scanty colloid; suggestive of a follicular neoplasm thyroid. Magnetic resonance imaging (MRI) showed a soft-tissue density mass lesion involving the entire sphenoid sinus, with sellar, suprasellar and parasellar extension (Figs 2 and 3) and a large heterogeneous mass left side of neck in left lower paracarotid region with central necrosis (Fig. 4). The patient was transferred to otorhinolaryngology department for further work-up. Otorhinolaryngological examination was within normal limits except for septal deviation to right side. We performed a diagnostic nasal endoscopy which revealed a polypoidal lesion covering the sphenoethmoidal recess. A wide sphenoidotomy was done and the sphenoid sinus was filled with a bleeding, friable, polypoidal mass from which biopsy was taken. Histopathologic examination of the specimen revealed metastasis from follicular carcinoma thyroid (Figs 5A and B). So, the final diagnosis was follicular carcinoma thyroid with sphenoid sinus metastasis. We planned for a total thyroidectomy followed by radioiodine ablation of the sphenoid secondaries. But unfortunately the patient disappeared and failed to turn up for further treatment.

\section{Case 2}

Our second patient is a 30 years old lady who presented with complaints of early morning headache in the right frontal region of 2 years, dimness of vision right eye of 1 month and diplopia on looking toward right of 1 month duration. She had no history of facial pain or numbness and had no other comorbidities. On examination, she had only perception of hand movements in the right eye and left eye showed 6/12 vision. On fundus examination, right optic atrophy was noted. She also had right lateral rectus palsy and paresthesia in the distribution of the maxillary

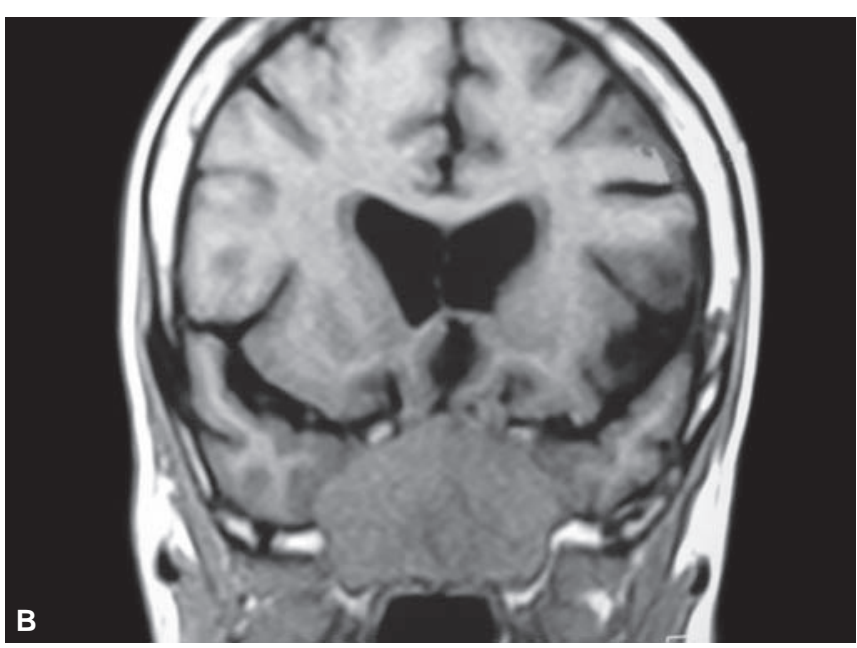

Figs 2A and B: (A) Sagittal and (B) axial MRI images showing a soft-tissue density mass lesion involving the entire sphenoid sinus, with sellar, suprasellar and parasellar extensions

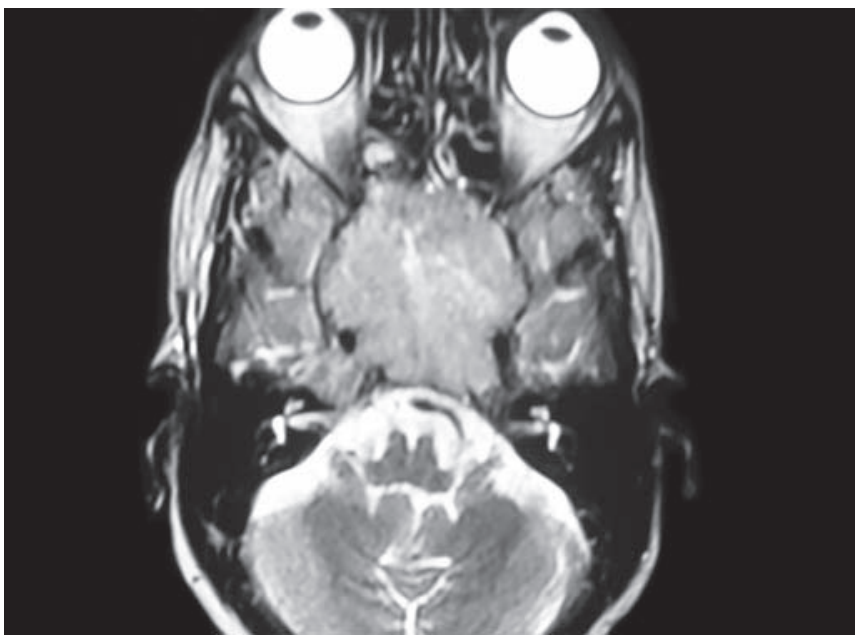

Fig. 3: Axial MRI image showing the mass lesion in the sphenoid sinus encasing the carotids

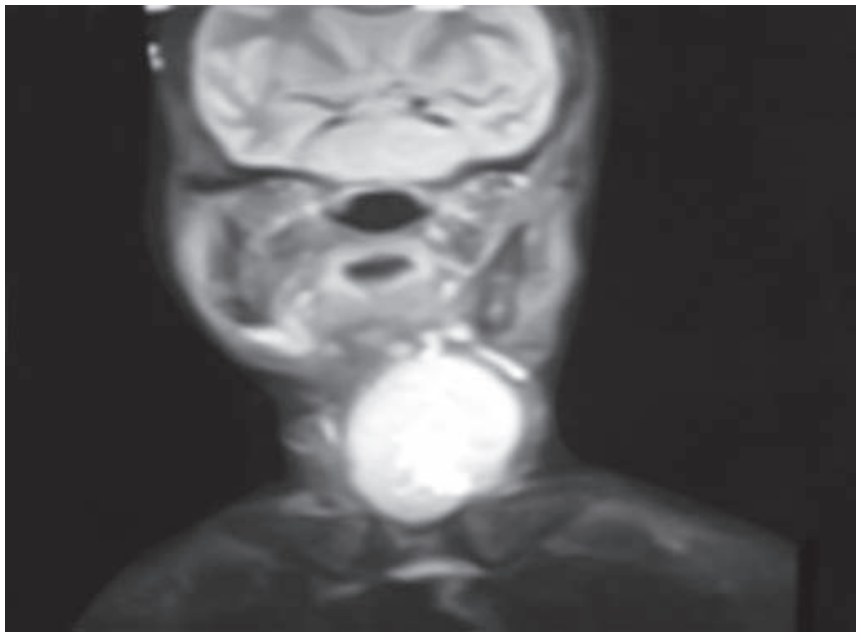

Fig. 4: Magnetic resonance imaging neck coronal view showing a large heterogeneous mass left side of neck in left lower para carotid region with central necrosis 

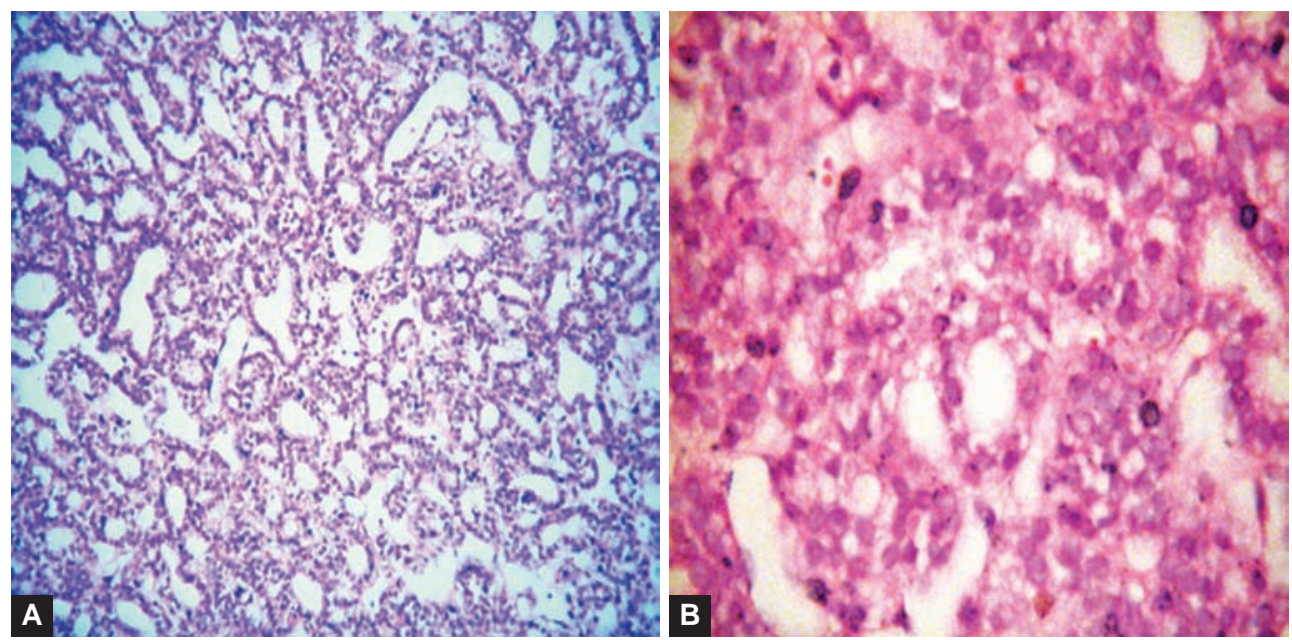

Figs 5A and B: (A) H\&E low and (B) high power view photomicrographs from sphenoidotomy specimen showing thyroid follicles lined by cuboidal cells displaying nuclear enlargement, overlapping and nuclear clearing

division of the right trigeminal nerve. Other cranial nerves were normal. Rest of the general examination was normal. We proceeded with radiological investigations. Computed tomography scan showed a large heterogenous

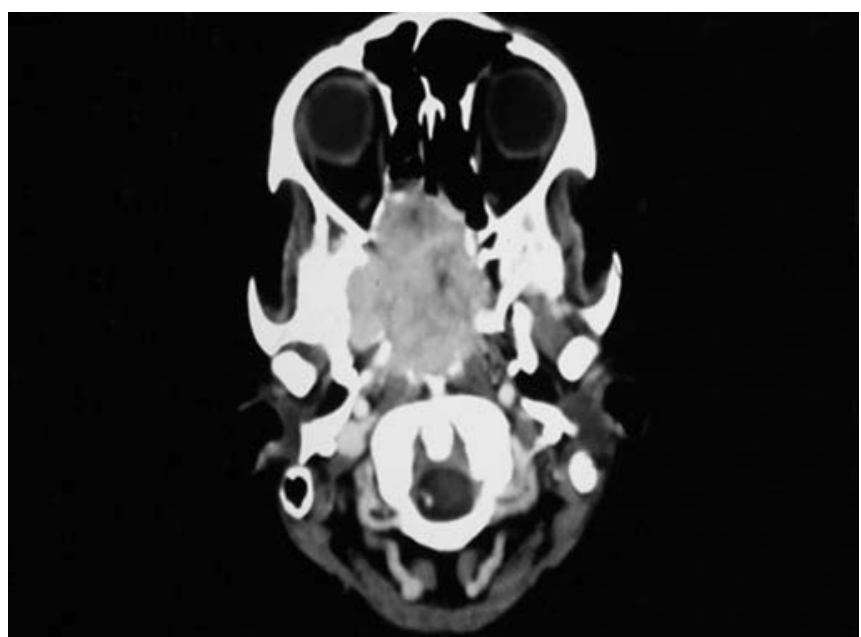

Fig. 6: Computed tomography image showing a large heterogenous lesion in the sphenoid sinus destroying the skull base lesion in the sphenoid sinus destroying the skull base (Fig. 6) and MRI brain showed the lesion extending into the orbit and compressing the right optic nerve (Figs 7A and $\mathrm{B})$. We then proceeded with a diagnostic nasal endoscopy and endoscopic transnasal, transsphenoidal decompression and excision of the mass. Biopsy was taken from the mass and sent for histopathological examination. Postoperative CT scan showed resolution of the mass lesion (Fig. 8). To our surprise the histopathology report came as metastasis from follicular carcinoma thyroid. However, this patient did not have an obvious thyroid swelling. Further investigations were planned but at the patient's request she was referred to Regional Cancer Centre, Thiruvananthapuram, Kerala, India for further management.

\section{DISCUSSION AND REVIEW OF LITERATURE}

Sinonasal tumors represent $1 \%$ of all cancers, ${ }^{9}$ and of these very few are metastatic. Renal primary tumors were most often responsible for secondary spread to the paranasal

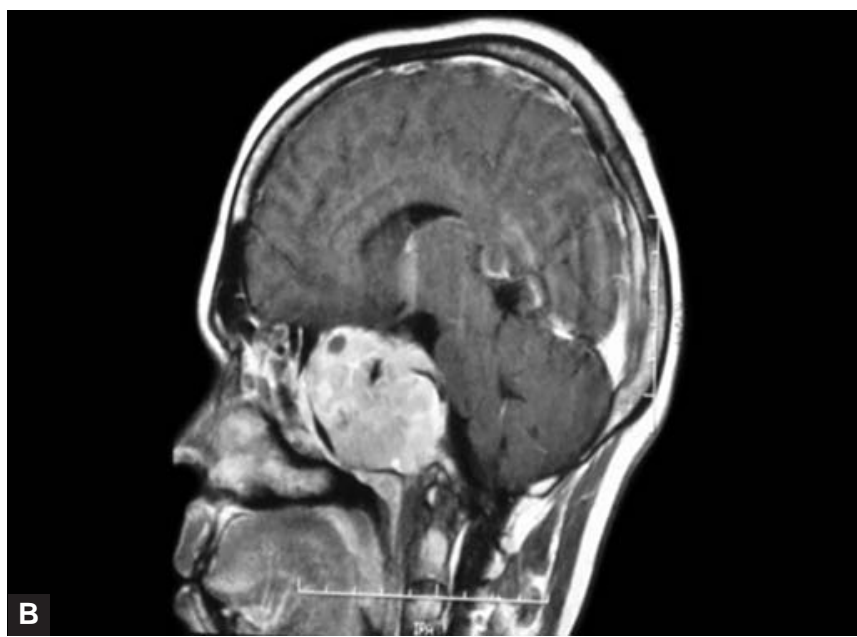

Figs 7A and B: (A) Axial and (B) sagittal MRI showing a large soft-tissue density lesion in the skull base extending into the orbit and compressing the right optic nerve with encasement of both carotids 


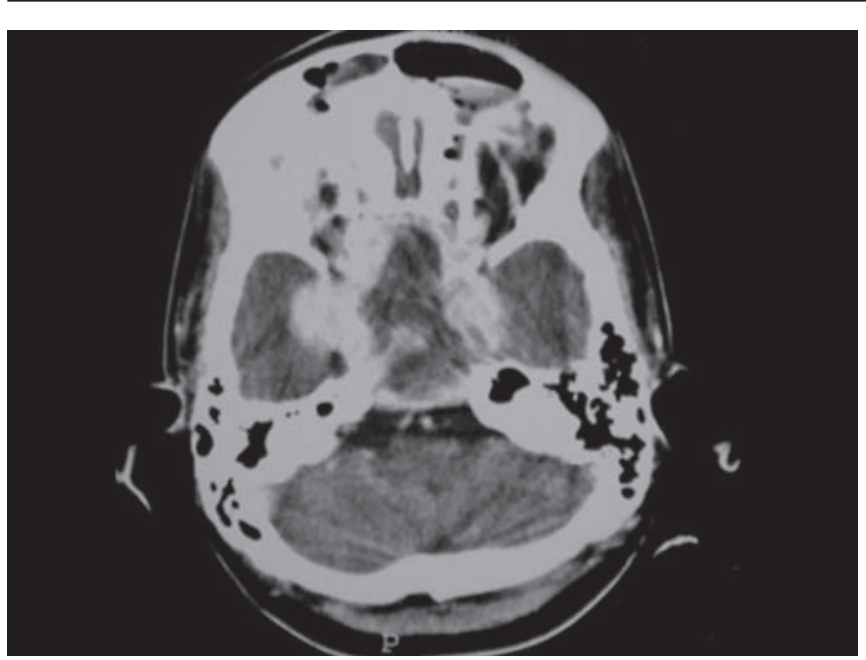

Fig. 8: Postoperative CT scan showing resolution of the mass lesion in the sphenoid sinus after endoscopic decompression

sinuses, ${ }^{10}$ and of these, the maxillary sinus is involved in $42 \%$, the nasal cavity in $42 \%$ and the sphenoid sinus less frequently. ${ }^{11}$ The sphenoid sinus is a very rare site of metastasis. ${ }^{4,12}$ Metastasis have been shown to occur even after the initial malignancy has been controlled. ${ }^{13}$

Differentiated carcinoma of the thyroid (DCT) accounts for $1 \%$ of visceral neoplasms. Annual incidence varies from 0.5 to 10 cases per 100,000 people. ${ }^{14}$ Papillary carcinoma of thyroid (PCT) is the most frequent type of DCT. Overall survival at 10 years is $95 \%$ in patients under 40 years of age and $75 \%$ in older patients. ${ }^{15}$ Despite the presence of distant metastases, the patients frequently survive for several years without treatment. Lung is the most common site of metastasis followed by bone. Incidence of paranasal sinus (PNS) metastasis is very low in case of thyroid carcinoma and only a handful of cases are reported. Only 12 cases of metastasis to the sphenoid sinus from thyroid malignancy has been reported so far and of these only six cases are from follicular carcinoma thyroid. ${ }^{5}$ Anatomically, the sphenoid sinus has important relations which dictate how tumors present. The cavernous sinus lies laterally containing the internal carotid artery, the occulomotor, trochlear and abducens nerves, and branches of the trigeminal nerve. The sella turcica is situated posterosuperiorly and contains the pituitary gland. Posteriorly, the sphenoid sinuses may about the brain stem. An isolated unilateral cranial nerve palsy is a 'red flag symptom' and mandatory urgent imaging is indicated. While CT scans are recognized as the modality of choice for imaging bone lesions, MRI is more reliable in detecting soft-tissue pathology. Thus, the scans are complimentary and should both be used and are essential for the diagnosis of metastases within the paranasal sinuses, as they reveal the size of the lesion and its extension to the adjacent structures, such as the orbit or the brain. There is not any specific radiological sign to differentiate metastases from a primary intracranial tumor.
Treatment of tumors invading the skull base from the sphenoid sinus is challenging. As malignancies of this nature carry a poor prognosis, in many cases treatment is restricted to symptomatic control, often through the use of palliative radiotherapy. Surgical resection may be possible, either via transfacial or lateral approaches, or via an endoscopic transphenoidal approach. ${ }^{16}$ All approaches carry significant risks including paralysis, damage to cranial nerves, and death as a result of injury to the internal carotid artery. Unresectable tumors may be treated with stereotactic radiotherapy. This treatment can be used in patients who have previously had radiation to the same area. Likely side effects include headache, nausea, vomiting and transient neurological symptoms secondary to cerebral edema.

\section{CONCLUSION}

We have presented these two cases to highlight the rare presentation of metastatic disease in the sphenoid sinus. Both our patients presented mainly with visual symptoms and there were no significant symptoms relating to the primary disease except for the long standing thyroid swelling as in the first patient. When present signs and symptoms relating to this are frequently the first presentation of disease and these patients should be vigorously evaluated for the possibility of primary as well as metastatic tumor of the sinus. Even though cure of patients with sphenoid sinus metastasis has not been reported, palliation with resolution of morbidity is possible.

\section{REFERENCES}

1. Abi-Fadel F, Smith PR, Ayaz A, Sundaram K. Paranasal sinus involvement in metastatic carcinoma. J Neurol Surg Rep 2012 Oct;73(1):57-59.

2. Maschka DA, Mcculloch TM, Nerad JA. Prostate cancer metastatic to the orbit. Annals Otol Rhinol Laryngol 1996; 105(1):70-71.

3. Cinberg JZ, Terrife D. Follicular adenocarcinoma of the thyroid in the maxillary sinus. Otolaryngol 1980;88(2):157-158.

4. Har-El G, Avidor I, Weisbord A, Sidi J. Carcinoma of the prostate metastatic to the maxillary antrum. Head and Neck Surg 1987;10(1):55-58.

5. Sachmechi I, Bitton RN, Sanelli-Russoand S, Thongpooswan S. An aggressive follicular variant of papillary thyroid carcinoma with unusual metastasis: a case report and review of literature BJMP 2014 Mar;7(1):705-709.

6. Mickel RA, Zimmerman MC. The sphenoid sinus: a site for metastasis. Otolaryngology Head and Neck Surg 1990 Jun; 102(6):709-716.

7. Lavasani L, Zapanta PE, Tanna N, Sadeghi N. Metastasis of prostatic adenocarcinoma to the sphenoid sinus. Annals of Otol Rhinol Laryngol 2006;115(9):690-693.

8. Patel M, Thinakkal R, Soboroff B, Borkenhagen R. Renal metastatic epistaxis: case report and update of literature, 1966-1978. Illinois Med J 1980;158(5):335-339. 
9. Tufano RP, Mokadam NA, Montone KT, Weinstein GS, Chalian AA, Wolf PF, et al. Malignant tumors of the nose and paranasal sinuses: hospital of the University of Pennsylvania experience 1990-1997. Am J Rhinol 1999;13(2): 117-123.

10. Bernstein JM, Montgomery WW, Balogh K. Metastatic tumors of the maxilla, nose and paranasal sinuses. Laryngoscope 1966;76:621-650.

11. Huang HH, Fang TJ, Chang PH, Lee TJ. Sinonasal metastatic tumors in Taiwan. Chang Gung Med J 2008;31(5):457-462.

12. Weig S, Howard J, Fry T. Metastatic laryngeal carcinoma as a cause of progressive ophthalmoplegia. Southern Med J 1989; 82(8):1051-1052.
13. Cumberworth V, Ohri A, Morrissey G, Stirling R. Late sinonasal metastasis from follicular thyroid carcinoma. J Laryngol Otol 1994;108:1010-1011.

14. Ferrer JC, Merino JF, Ponce JL, Pinon F. Unusual metastasis of differentiated thyroid carcinoma. An Med Interna 2002;19: 579-582.

15. Vázquez AS, Hernández LC, Muñiz MÁ. Metastases in the sphenoidal sinus in a patient with papillary thyroid cancer. Clin Transl Oncol 2005;7:324-327.

16. Lund V, Stammberger H, Nicolai P, Castelnuovo P. European position paper on endoscopic management of tumors of the nose, paranasal sinuses and skull base. Rhinol 2010;48(2):s22. 1001-1144. 